\title{
Analysis of Duopoly Price Competition between WLAN Providers
}

\author{
Zhen Kong \\ Department of EEE \\ Univ. of Hong Kong \\ Hong Kong, CHINA \\ zkong@eee.hku.hk
}

\author{
Bruno Tuffin \\ INRIA Rennes \\ Campus Univ. de Beaulieu \\ Rennes, FRANCE \\ btuffin@irisa.fr
}

\author{
Yu-Kwong Kwok \\ Department of ECE \\ Colorado State Univ. \\ Fort Collins, CO, USA \\ ricky.kwok@colostate.edu
}

\author{
Jiangzhou Wang \\ Department of Electronics \\ University of Kent \\ Canterbury, Kent, UK \\ j.z.wang@kent.ac.uk
}

\begin{abstract}
With the rapid development of wireless Internet services, several WLAN service providers may coexist in one public hotspot to compete for the same group of customers, leading to an inevitable price competition. The charged price and the provisioned packet loss at each provider are major factors in determining users' demands and behaviors, which in turn will affect providers' revenue and social welfare.

In this paper, we set up a novel game model to analyze a duopoly price competition. We first show the users' demands are distributed between providers according to a Wardrop Equilibrium and then prove the existence of a Nash equilibrium on providers' charged prices. Through analysis, we further find that in Nash equilibrium state the social welfare is very close to its maximal value in cooperative situation. Furthermore, the providers' aggregate revenues also do not decrease when the users have high sensitivity about the charged prices. Thus the competitive duopoly WLAN market can still run in an efficient way even in the absence of complex regulation schemes.
\end{abstract}

Keywords: Price competition; Price of anarchy; Wardrop equilibrium; Nash equilibrium; WLAN; hotspot.

\section{INTRODUCTION}

Significant investments in broadband wireless networks are proliferating around the world in recent years, and have led to a highly competitive environment for wireless LAN (WLAN) service providers. They have to compete with each other and attract users in order to generate profits. In a typical competitive hotspot environment (e.g., a public cyber-café), several WLAN providers may coexist to provide wireless access services for the same group of users. In attempting to attract users and optimize their revenues, they need to price their services by taking into account a wide range of factors, including preferences of end-users, the quality-of-service (QoS) limitations of used technology, and the potential competition from other providers. Furthermore, when the QoS and prices are observed, wireless users will allocate their demands across the providers. This process will in turn affect the revenue of providers. Thus, with the capability to affect users' demand and providers' revenue, pricing in wireless networks has gained much attention recently [1]

Studying the impact of price competition is receiving increasing attention in the networking community. For general ideas on pricing, the reader is referred to [1]. The pricing game among wireless providers with fixed capacity is analyzed in [2], and it is shown that the price of anarchy (PoA) [3] is 1. Acemoglu et al. [4] study a pricing problem in which the users are sensitive to the price and a convex congestion delay, and give tight bounds on PoA. In [5] Hayrapetyan et al. gives an improved bound for the special case in which packet delay is a pure negative effect, which is linear or convex. Niyato et al. [6] investigate two levels of competitions in cognitive wireless mesh network, and propose game theoretic solutions to choose price and transmission rate for primary users and secondary users, but the demand distribution of secondary users is not analyzed.
In [7] a cost-price mechanism based on users' channel occupancy is proposed in WLAN to maximize the system throughput, but the competition between different WLAN providers is not analyzed. Furthermore, because WLAN standard employs a contention based random multiple access method based on CSMA/CA, i.e. DCF, as the fundamental MAC technique, there exists packet collision, which will result in packet loss. On the other hand, wireless transmission error may lead to packet loss too. Thus, in this paper, we consider packet loss rate (PLR) instead of delay or rate as negative externality [1], and find that it is indeed concave other than convex in WLAN environment. Thus the previous finding cannot be used in WLAN situations directly. To the best of our knowledge, the problem of pricing competition for WLAN providers with the consideration of price and PLR is relatively unexplored.

In this paper, we focus on a duopoly competition, in which two WLAN providers compete for a same group of wireless users by adjusting the price charged. The provider's aim is to maximize its own profit, while the users, driven by self-interests, rationally choose the service provider offering the best combination of QoS and price.

We assume that users are sensitive to the experienced PLR as well as charged prices. The PLR at each access point (AP) occurs because of packets collision or packet transmission error. Generally, the users are more likely to connect to the provider with good channel condition and/or low price, but when more users connect to a provider, the probability of collision will increase too. This feature is also known as negative externality [1], since the decision made by a user will have a negative effect on the payoff of others connected to the same provider. Thus, it can affect user's willingness to accept a provider's service.

Consequently, each user has to calculate the experienced cost to choose a provider according to the charged price and estimated PLR. We assume each user likes to choose the provider with the minimum experienced cost and the number of users (i.e., demand) decreases with the cost. This leads us to research some important questions: how the users' demand is split among providers when their price strategies are given and the PLRs are estimated? Does there exist Nash equilibrium (NE) [9] price vector, under which no provider can unilaterally improve its own revenue by changing its price? Further, whether there exists the efficiency loss, i.e., PoA, as what is shown by many prior works, e.g., [4], [5]?

To answer these questions, we first set up a game model to analyze negative externalities induced by PLR, give its approximation and find it is concave indeed. Then we characterize the Wardrop equilibrium (WE) [8] for the distribution of users' demands. After that, we prove the existence of NE on providers' charged prices. Then through numerical analysis we find that PoA can be close to 1.1, which indicates that the social welfare does not suffer too much in this competitive environment. Furthermore, we find 
that the competition does not lead to revenue loss when the users are very sensitive to charged prices.

The rest of this paper is organized as follows. We present the system model in Section II. Section III analyses the demand distribution in terms of WE. The pricing game is researched in Section IV. Section V gives numerical analysis of efficiency and we conclude in Section VI.

\section{MODEL}

\section{A. Network model}

We consider an IEEE 802.11 hotspot area covered by 2 APs, and let $\Omega=\{1,2\}$ be the set of APs.- Each AP $i \in \Omega$ is controlled by a distinct provider, which charges a price $p_{i}$ per user for using its service. Thus here the terms of AP and provider are interchangeable. We assume APs share the same frequency bandwidth in the network, whereas different APs are being operated on different frequency channels and using different PHY mode. Thus there is no interference among different users. DCF is used as MAC level multiuser access method. We also ignore hidden node problem, so that every user can sense all other's transmission as in [10]. Furthermore we operate in saturation conditions, i.e., the transmission queue of each user is assumed to be always nonempty. And there is only one user that can transmit its packets successfully to the belonged AP at one time slot.

Let $x_{i}$ be the number of users connected to AP $i$. Note that users are assumed to be infinitesimal, each of them having a negligible impact on others. We assume that when the $x_{i}^{\text {th }}$ user connects to the AP $i$, it will get a utility of $U_{i}\left(x_{i}\right)$, which is its willingness to pay to get service. Similar to what is done in [4], we use the assumption that when a user decides to receive the WLAN access service, it will get a reservation preference of $R$, i.e. $U_{i}\left(x_{i}\right)=R$. But a cost $c_{i}\left(x_{i}\right)$ will also be experienced when there are $x_{i}$ users, which is directly related with charged price $p_{i}$ and packet loss rate $P L R_{i}\left(x_{i}\right)$. Furthermore, this user will not connect to AP $i$ if the experienced cost exceeds its reservation preference. Then the aggregate user's surplus in AP $i$ can be

$$
S_{i}=\left(R-c_{i}\left(x_{i}\right)\right) \cdot x_{i}
$$

where the cost $c_{i}\left(x_{i}\right)$ can be expressed as

$$
c_{i}\left(x_{i}\right)=p_{i}+f_{i}\left(P L R_{i}\left(x_{i}\right)\right)=p_{i}+f_{i}\left(x_{i}\right)
$$

where $f_{i}(\cdot)$ is used to express the experienced cost resulting from PLR. For the sake of simplicity, we assume users are homogeneous and let $f_{i}\left(x_{i}\right)=\alpha \cdot P L R_{i}\left(x_{i}\right)$, where $\alpha \geq 0$. The experienced cost is an important parameter since users' demand and providers' revenue are highly related to it.

In this work, we define the revenue of provider $i$ as

$$
\Pi_{i}\left(p_{i}\right)=p_{i} \cdot x_{i}
$$

As defined in [4], the social welfare (SW) can be expressed by adding the utilities of all users and providers, i.e.

$$
S W=\sum_{i \in \Omega}\left(R-f_{i}\left(x_{i}\right)\right) \cdot x_{i}
$$

\section{B. Packet loss rate}

In 802.11 systems, PLR can be expressed as

$$
P L R_{i}\left(x_{i}\right)=P_{i}^{l}=1-\left(1-P_{i}^{c}\right)\left(1-P_{i}^{t}\right)=P_{i}^{c}+P_{i}^{t}-P_{i}^{c} \cdot P_{i}^{t}
$$

where $P_{i}^{l}$ and $P_{i}^{c}$ are packet loss rate and packet collision rate experienced at AP $i$ with $x_{i}$ users, respectively, and $P_{i}^{t}$ is the minimal packet transmission error rate supported by AP $i$.

In fact, due to location-dependent characteristics, the transmission error rate will be different for different users. To focus on the price setting for different providers and ease the analysis, the exogenous information used in this game is the minimal transmission error rate $P_{i}^{t}$ that AP $i$ can support under its geography situation and provisioned transmission techniques, e.g. coding and modulation. Furthermore, a user may also automatically move to a satisfactory place for an acceptable transmission condition. Then because there is no interference between providers in this model, we can assume that $P_{i}^{t}$ at a particular provider is maintained as a constant value and different providers will support different $P_{i}^{t}$.

In terms of packet collision rate $P_{i}^{c}$, here we refer to it as the packet collision observed at each individual user [10], i.e. it is the probability that one user encounters collisions when it transmits packets. Define $P_{i}^{0}$ as the transmission probability for each user. Then if $x_{i}$ users are connected to the provider $i$, we have $P_{i}^{c}=1-\left(1-P_{i}^{0}\right)^{x_{i}-1}$. Since the providers want to maximize their profits, the assumption of maximum saturation throughput is reasonable in this research. In [10], an approximation for $P_{i}^{0}$ under maximum achievable saturation throughput is given by

$$
P_{i}^{0}=\frac{\sqrt{\left[x_{i}+2\left(x_{i}-1\right)\left(T_{c}^{*}-1\right)\right] / x_{i}-1}}{\left(x_{i}-1\right)\left(T_{c}^{*}-1\right)} \approx \frac{1}{x_{i} \sqrt{T_{c}^{*} / 2}}=\frac{1}{x_{i} K}
$$

where $K=\sqrt{T_{c}^{*} / 2}$, and $T_{c}^{*}$ is the average time when the channel is sensed busy by each station during a collision, which is determined as a constant by given PHY and MAC mechanism. Thus we have

$$
\begin{aligned}
& \operatorname{PLR}_{i}\left(x_{i}\right)=P_{i}^{l}=1-\left(1-P_{i}^{c}\right)\left(1-P_{i}^{t}\right)=1-\left(1-\frac{1}{x_{i} K}\right)^{x_{i}-1}\left(1-P_{i}^{t}\right) \\
& =1-\left(1-P_{i}^{t}\right) \cdot \mathrm{e}^{\left(x_{i}-1\right) \cdot \ln \left(1-\frac{1}{x_{i} K}\right)} \approx 1-\left(1-P_{i}^{t}\right) \cdot e^{\left(x_{i}-1\right)\left[\frac{-1}{x_{i} K}+\frac{1}{2\left(x_{i} K\right)^{2}}\right]} \\
& \approx 1-e^{\frac{-1}{K}} \cdot\left(1-P_{i}^{t}\right) \cdot e^{\frac{1}{x_{i}} \cdot\left(\frac{1}{K}+\frac{1}{2 K^{2}}\right)}
\end{aligned}
$$

We can further yield

$$
x_{i}=P L R_{i}^{-1}\left(P_{i}^{l}\right)=\frac{v}{\ln \left(1-P_{i}^{l}\right)+\omega_{i}}
$$

where $\omega_{i}=\frac{1}{K}-\ln \left(1-P_{i}^{t}\right), v=\frac{1}{K}+\frac{1}{2 K^{2}}$. Remark that $P L R_{i}\left(x_{i}\right)$ is strictly increasing with regard to $x_{i}$ and $P_{i}^{t}$. Also, since the second derivative $P L R_{i}^{\prime \prime}\left(x_{i}\right)<0$, it's concave.

\section{Distribution OF Users' DEMAND}

When a user wants to access to a WLAN, it will face several competitive providers with different charged prices and provisioned QoS, and therefore feels different experienced $\operatorname{costs} c_{i}\left(x_{i}\right)$. Naturally it prefers to choose the provider with the minimum cost $\underline{C}=\min \left(c_{i}\left(x_{i}\right)\right)$. 
Furthermore, if a provider's cost is too high, there will no additional user willing to choose it, and even already connected ones will be expected to switch. Then at equilibrium state, the experienced cost will be identical at all providers having a positive demand, or the number of users connected to one provider will be zero because of a too high access price. This is also known as Wardrop equilibrium (WE), which can be mathematically defined as follows.

Definition 1: The users demand $X^{W E}=\left(x_{1}, x_{2}\right)$ is said to achieve WE if

$x_{i} \cdot\left(c_{i}\left(x_{i}\right)-\underline{C}\right)=0 \quad \forall i \in \Omega$, and $\underline{C}=\min _{i \in \Omega}\left(C_{i}\left(x_{i}\right)\right), x_{i}>0$

Also the total level of demand verifies $\sum_{i \in \Omega} x_{i}=X(\underline{C})$.

This equation means that the providers with positive demand will have the same experienced $\cos t \underline{C}$; otherwise the provider will have zero demand due to the high cost. Since $c_{i}\left(x_{i}\right)-\underline{C} \geq 0$ and $\sum_{i \in \Omega} x_{i}=X(\underline{C})$, the existence of WE in our model can be verified as that in [4], [8]. Next we will derive the uniqueness of WE in this research.

We assume the total number of users in the system decreases with the minimum experienced cost. Then we can express the aggregate demand function $X(\underline{C})$ as

$$
X(\underline{C})=X_{0}-d \cdot \underline{C}, \text { for } d \geq 0
$$

where $d$ is a demand parameter to express user's sensitivity to the experienced cost. Then we can characterize the WE vector $X^{W E}=\left(x_{1}, x_{2}\right)$ as follows.

Proposition 1: With demand function $X(\underline{C})$ and price vector $P=\left(p_{1}, p_{2}\right)$, WE vector $X^{W E}=\left(x_{1}, x_{2}\right)$ can be characterized as the smallest solution of the inequation $X(\underline{C}) \leq \sum_{i \in \Omega} x_{i}(\underline{C})$ with $\underline{\mathrm{C}} \in\left[0, X^{-1}\left(X_{0}\right)\right]$, and

$$
x_{i}=\left\{\begin{array}{cc}
X(\underline{C}) & \frac{\underline{C}-p_{i}}{\alpha} \geq P L R_{i}\left(X_{0}\right) \\
P L R_{i}^{-1}\left(\frac{\underline{C}-p_{i}}{\alpha}\right) & P L R_{i}\left(X_{0}\right)>\frac{\underline{C}-p_{i}}{\alpha}>P L R_{i}(0) \\
0 & \frac{\underline{C}-p_{i}}{\alpha} \leq P L R_{i}(0)
\end{array}\right.
$$

Proof: Since in WE state, $\underline{C}=p_{i}+f_{i}\left(x_{i}\right)$ for $\forall x_{i}>0$, and the total demand limit is $X_{0}$, we have $x_{i}=P L R_{i}^{-1}\left(\frac{\underline{C}-p_{i}}{\alpha}\right)$, when $P L R_{i}\left(X_{0}\right)>\frac{\underline{C}-p_{i}}{\alpha}>P L R_{i}(0)$. Otherwise when $p_{i}$ is too high, and $\frac{\underline{C}-p_{i}}{\alpha} \leq P L R_{i}(0)$, we can get $x_{i}=0$; and when $p_{i}$ is too small, and all users want to connect with it, we have $x_{i}=X(\underline{C})$. Then we can get $x_{i}(\underline{C})$ as the expression in (10). What's more, after one user choose a provider, the minimum experienced cost $\underline{C}=\min \left(c_{i}\left(x_{i}\right)\right)$ increases and $X(\underline{C})$ is decreased. If $X(\underline{C})>\sum_{i} x_{i}(\underline{C})$, new user can enter the system to choose a provider; otherwise, the demand limit is reached, and the corresponding WE is achieved too.

\section{PRICE COMPETITION ANALYSIS}

In this duopoly environment, each provider seeks to set its price in order to maximize its own revenue. Thus we set up a price game and the set of providers $\Omega$ represents the players. For provider $i \subset \Omega=\{1,2\}$, its action is defined as the price choice $p_{i}$.Then the action profile is denoted as the price vector $p=\left(p_{1}, p_{2}\right)$. Its utility is expressed by the revenue $\Pi_{i}\left(p_{i}, p_{-i}\right)$, where $p_{-i}$ denotes the prices chosen by providers else other than $i$. Then we define NE as

Definition 2: A price vector $p^{N E}=\left(p_{1}^{N E}, p_{2}^{N E}\right)$ is said to be a Nash equilibrium if for all $i \in \Omega$,

$$
\Pi_{i}\left(p_{i}^{N E}, p_{-i}^{N E}\right) \geq \Pi_{i}\left(p_{i}, p_{-i}^{N E}\right) \forall p_{i}>0
$$

We first prove a lemma that establishes some results with a price configuration $p$.

Lemma 1: Assume that both providers have positive demand $x=\left(x_{1}, x_{2}\right)$ for price $p=\left(p_{1}, p_{2}\right)$. If provider $i$ decreases its price to $p_{i}^{n}=p_{i}-\varepsilon<p_{i}$, and $p_{j}$ for provider $j \neq i$ is unchanged, where superscript " $n$ " is used to refer the values corresponding to a new situation; then

(1) the PLR increases to $P_{i}^{l, n}>P_{i}^{l}$

(2) the least experienced cost decreases to $\underline{C}^{n}=\underline{C}-\phi<\underline{C}$, and $\phi<\varepsilon$, where $\phi>0, \varepsilon>0$.

Proof: Since $p_{i}^{n}<p_{i}$ and $p_{j}^{n}=p_{j}$, more users will switch from provider $j$ to $i$. Thus $x_{i}^{n}>x_{i}$ and $x_{j}^{n}<x_{j}$. Since PLR is increasing related to user number, we have $P_{i}^{l, n}>P_{i}^{l}$. Then

$$
\begin{aligned}
& \underline{C}^{n}=\min _{i \in \Gamma}\left(c_{i}^{n}\left(x_{i}^{n}\right)\right) \leq c_{j}^{n}\left(x_{j}^{n}\right)=p_{j}+f_{j}\left(x_{j}^{n}\right) \\
& <p_{j}+f_{j}\left(x_{j}\right)=c_{j}\left(x_{j}\right)=\underline{C}
\end{aligned}
$$

Let $p_{i}^{n}=p_{i}-\varepsilon$ and $\underline{C}^{n}=\underline{C}-\phi$, then we get

$$
\begin{aligned}
& \underline{C}^{n}=\underline{C}-\phi=p_{i}^{n}+f_{i}\left(x_{i}^{n}\right)=p_{i}-\varepsilon+f_{i}\left(x_{i}^{n}\right) \\
& =p_{i}-\varepsilon+\left(1-e^{-\omega_{i}} \cdot e^{\frac{v}{x_{i}}}\right)+\left(e^{-\omega_{i}} \cdot e^{\frac{v}{x_{i}}}-e^{-\omega_{i}} \cdot e^{\frac{v}{x_{i}^{n}}}\right) \\
& =\underline{C}-\varepsilon+\left(e^{-\omega_{i}} \cdot e^{\frac{v}{x_{i}}}-e^{-\omega_{i}} \cdot e^{\frac{v}{x_{i}^{n}}}\right)>\underline{C}-\mathcal{E}
\end{aligned}
$$

Thus $\phi<\varepsilon$.

With this observation, we can show the existence of NE. Proposition 2: When packet loss rate is much smaller than 1, there exists a price vector $P^{N E}=\left(p_{1}^{N E}, p_{2}^{N E}\right)$ to achieve Nash equilibrium in this price competition.

Proof: Without loss of generality, consider a possible change of provider 1 from $p_{1}^{N E}$ to $p_{1}^{n}=p_{1}^{N E}-\varepsilon<p_{1}^{N E}$. Then $P_{i}^{l, n}>P_{i}^{l, N E}$, we can have

$$
\begin{aligned}
& \Pi_{1}^{n}-\Pi_{1}^{N E}=p_{1}^{n} \cdot x_{1}^{n}-p_{1}^{N E} \cdot x_{1}^{N E} \\
& =\left(p_{1}^{N E}-\varepsilon\right) \cdot \frac{v}{\omega_{i}+\ln \left(1-P_{i}^{l, n}\right)}-p_{1}^{N E} \cdot x_{1}^{N E}
\end{aligned}
$$

Since $P_{i}^{l, n} \ll 1$, with Taylor expansion, we have 


$$
\begin{aligned}
& \Pi_{1}^{n}-\Pi_{1}^{N E} \approx\left(p_{1}^{N E}-\varepsilon\right) \cdot \frac{v}{\omega_{i}-P_{i}^{l, n}}-p_{1}^{N E} \cdot x_{1}^{N E} \\
& <\left(p_{1}^{N E}-\varepsilon\right) \cdot \frac{v}{\omega_{i}-P_{i}^{l, N E}}-p_{1}^{N E} \cdot x_{1}^{N E} \\
& \approx\left(p_{1}^{N E}-\varepsilon\right) \cdot x_{1}^{N E}-p_{1}^{N E} \cdot x_{1}^{N E}<0
\end{aligned}
$$

With this price setting, no provider can improve its revenue by unilaterally changing its price, thus resulting in a NE.

\section{EFFICIENCY ANALYSIS}

We investigate the efficiency of this game in terms of social welfare and providers' revenue. Here we use PoA as a measure of the worst case difference between the social welfare of a cooperatively optimal solution (social optimum) and that in a non-cooperative NE state. Similar to that in [5], we define PoA as follows.

Definition 4: If $P^{S}=\left(p_{1}^{S}, p_{2}^{S}\right)$ is the price vector that maximizes social welfare, $P^{N E}=\left(p_{1}^{N E}, p_{2}^{N E}\right)$ is the price vector in NE state; then PoA is defined as $P o A=\frac{S W\left(P^{S}\right)}{S W\left(P^{N E}\right)}$.

Since $S W\left(P^{S}\right) \geq S W\left(P^{N E}\right)$, we get $P o A \geq 1$. When PoA is close to 1 , it means the social welfare arrived at competitive environment is nearly as good as that reached through cooperative optimization. Whereas a large PoA means the competition is less efficient.

In non-cooperative situation, we can first get NE price vector $P^{N E}$ by finding the intersection of both users' best response functions as introduced in [9], and then find the corresponding WE distribution $X^{W E}$ with Proposition 1. After that we can calculate $S W\left(P^{N E}\right)$ through $P^{N E}$ and $X^{W E}$ directly. While for $S W\left(P^{S}\right)$ in cooperative situation, it can be found by solving the following optimization problem.

$$
S W\left(P^{S}\right)=\max \sum_{i \in \Gamma}\left(R-f_{i}\left(x_{i}\right)\right) \cdot x_{i}
$$

subject to:

$$
\begin{gathered}
x_{i} \cdot\left(p_{i}+f_{i}\left(x_{i}\right)-\underline{C}\right)=0 \\
\sum_{i \in \Gamma} x_{i} \leq X(\underline{C})
\end{gathered}
$$

where the constraint I means the solution should satisfy WE; and the constraint II is used to constrain the user demand. Since the above problem has a continuous objective function and a compact constraint set, the existence of a social optimum is guaranteed.

Based on above analysis, we can investigate the PoA through numerical methods with two APs that either compete or cooperate with each other, in which the total number of users $X_{0}$ is 20 and $R$ is 1 . We also set $K=9.334$ as that in [10].

In Figure 1, we show the best response under different set of prices. Both of the packet transmission error rates for these two providers are set to be 0.01 , and demand parameter is 10 . We find that there exist a unique NE at prices $(0.4,0.4)$. A similar result with $\mathrm{NE}$ at prices $(0.32,0.22)$ also exist in Figure 2, where $P_{1}^{t}=0.01$ and $P_{2}^{t}=0.03$, and $d=3$. These verify the existence of NE under different situations.

Fig. 3 and Fig. 4 show the social welfare under different transmission error rate and demand parameter combinations.
We can see that SW increases with demand parameter, while in NE state it is close to that in cooperative situations. From Fig.5 and Fig.6, the PoA is near 1.1. Then letting the providers compete with each other will yield almost the same social welfare as a global market regulator would have given. Thus this competition is not as inefficient as what we usually look in a non-cooperative situation.

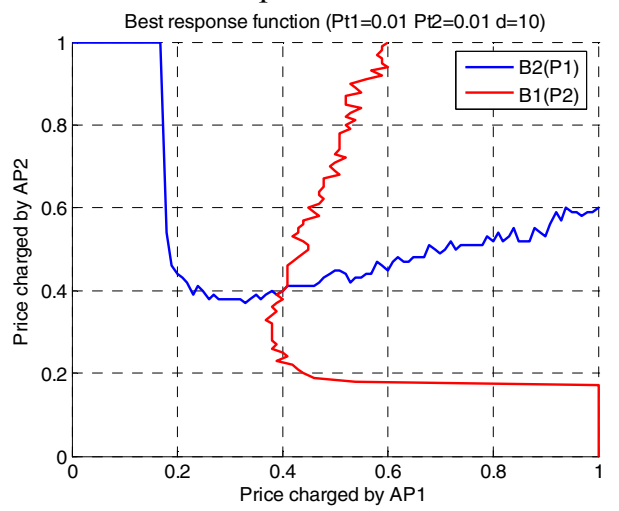

Fig. 1: Best response function $\left(P_{1}^{t}=P_{2}^{t}=0.01, d=10\right)$.

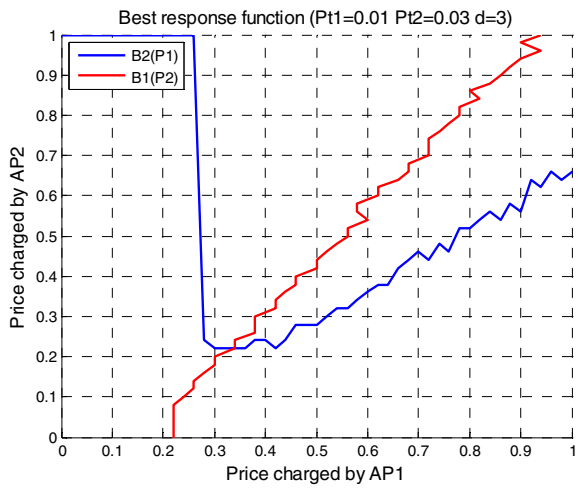

Fig. 2: Best response function $\left(P_{1}^{t}=0.01, P_{2}^{t}=0.03, d=3\right)$.

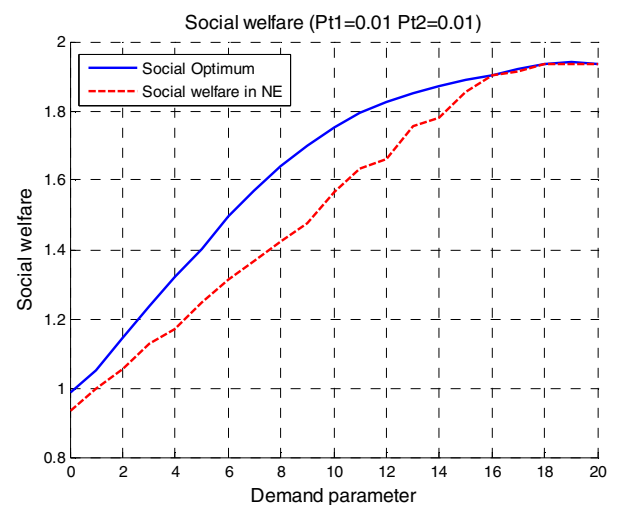

Fig. 3: SW vs. Demand parameter $\left(P_{1}^{t}=P_{2}^{t}=0.01\right)$.

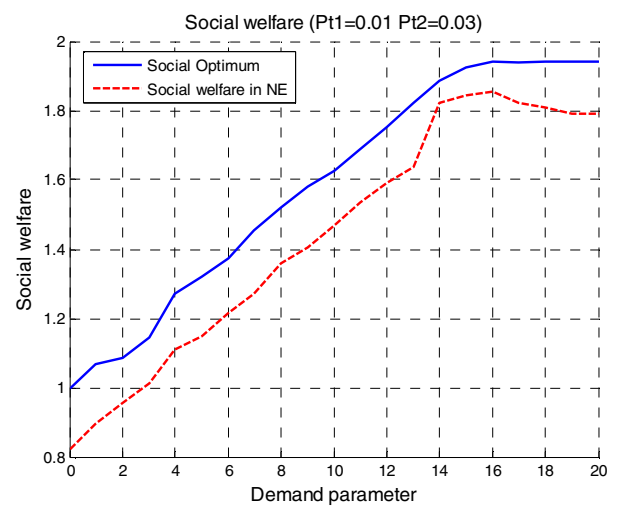

Fig. 4: SW vs. Demand parameter $\left(P_{1}^{t}=0.01, P_{2}^{t}=0.03\right)$. 


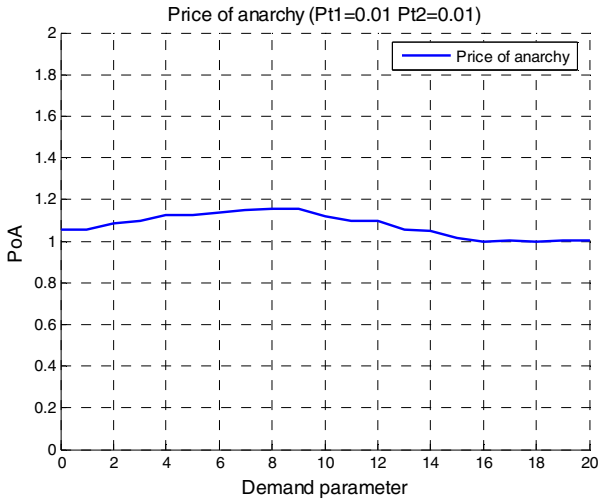

Fig. 5: PoA vs. Demand parameter $\left(P_{1}^{t}=P_{2}^{t}=0.01\right)$.

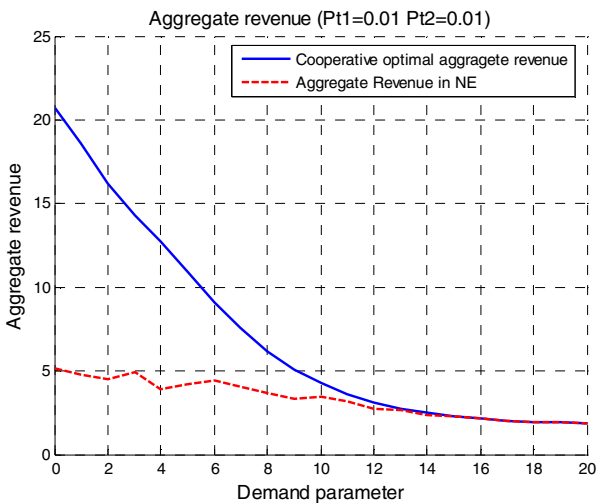

Fig. 7: Aggregate revenue vs. Demand parameter

$$
\left(P_{1}^{t}=P_{2}^{t}=0.01\right) \text {. }
$$

Though the efficiency of SW is not decreased much under competition, the providers' revenue in NE state is much smaller than that in cooperative situations when the demand parameter is small as shown in Fig.7 and Fig, 8. But when the demand parameter increases, the aggregate revenues in NE state will eventually approach to the cooperative maximal revenue. This is because the larger the demand parameter, the more sensitively that the user demand responds to the change of prices. If the user is not sensitive about the price, the providers would like to cooperate or even collude with each other to gain high revenue by setting high price. But when the user has higher price sensitivity, it will not receive any providers' service at all if the charged prices are too high. Then the providers will be more rational and prefer to set relatively lower prices so as to attract users. Though the revenue is decreased with demand parameter, it will consequently be same as cooperative maximal revenue. Furthermore, as shown in Fig. 3 and Fig.4, the social welfare also improves with the increasing of user's price sensitivity. Usually the users are very sensitive to the price of wireless services, thus all entities including providers and users will not suffer from this competition.

\section{CONCLUSIONS}

In this paper, the impact of charged price and the provisioned PLR by different WLAN providers on users' demand, providers' revenue and social welfare in a duopoly environment is investigated. Based on a game-theoretic model, we first analyzed the negative externalities associated with PLR. Then we found the existence and uniqueness of a WE for users' demand distribution between providers, and determined the existence of the NE of price competition. Furthermore, through numerical analysis, we showed that the social welfare will not suffer too much

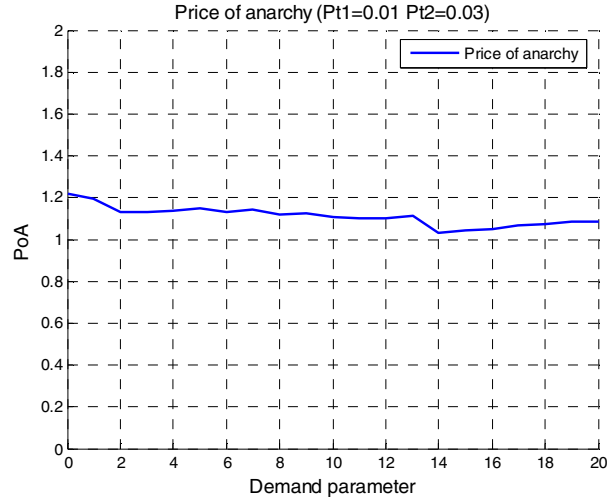

Fig. 6: PoA vs. Demand parameter $\left(P_{1}^{t}=0.01, P_{2}^{t}=0.03\right)$.

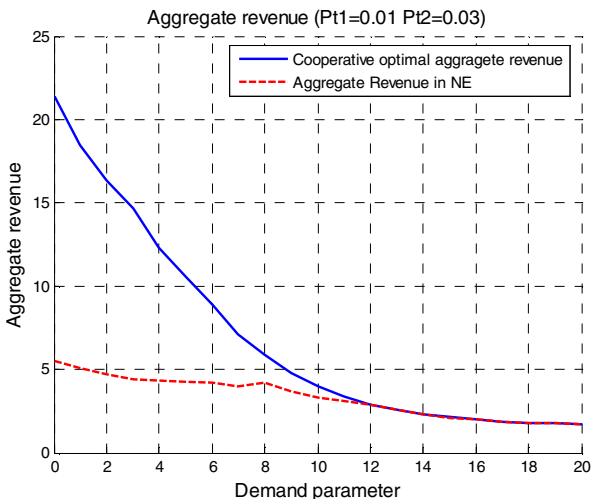

Fig. 8: Aggregate revenue vs. Demand parameter

$$
\left(P_{1}^{t}=0.01, P_{2}^{t}=0.03\right) \text {. }
$$

under price competition. Thus even without administrative enforcement, the competitive market itself can still determine the right price without degrading social welfare or even providers' revenues, especially when the users are very sensitive to the price (In fact this is just the normal case for user's attitude on price for wireless services). In the next step, we want to improve the system model and study this competition in a more realistic setting. Another interesting issue concerns the competition in a more general oligopoly environment, where there exist more than two competitive providers.

\section{REFERENCES}

[1] C. Courcoubetis and R. Weber, Pricing Communication Networks: Economics, Technology and Modelling, John Wiley \& Sons, 2003

[2] P. Maille and B. Tuffin, "Analysis of Price Competition in a Slotted Resource Allocation Game," In Proc. IEEE INFOCOM 2008, Phoenix, USA, Apr. 2008.

[3] C. Papadimitriou, "Algorithms, Games, and the Internet," In Proc. ACM STOC 2001, pp. 749-753, Hersonissos, Greece, July 2001.

[4] D. Acemoglu and A. Ozdaglar, "Competition and Efficiency in Congested Markets," Mathematics of Operations Research, vol. 32, no.1, pp. 1-31, Feb. 2007.

[5] A. Hayrapetyan, E. Tardos and T. Wexler, "A Network Pricing Game for Selfish Traffic," In Proc. ACM PODC 2005, pp. 284-291, Las Vegas, NV, USA, July 2005.

[6] D. Niyato, E. Hossain, and L. Le, "Competitive Spectrum Sharing and Pricing in Cognitive Wireless Mesh Networks," in Proc. IEEE WCNC 2008, pp. 1431-1435, Las Vegas, NV, USA, Mar.-Apr. 2008.

[7] S. Shakkottai, E. Altman, and A. Kumar, "Multihoming of Users to Access Points in WLANs: A Population Game Perspective," IEEE J. Select. Areas Commun., vol. 25, no. 6, pp.1207-1215, Aug. 2007.

[8] J. G. Wardrop, "Some Theoretical Aspects of Road Traffic Research," Proceedings of the Institute of Civil Engineers, Part II, vol. 1, pp. 325-378, London, UK, 1952.

[9] M. J. Osborne and A. Rubinstein, A Course in Game Theory, MIT Press, 1994.

[10] G. Bianchi, "Performance Analysis of the IEEE 802.11 Distributed Coordination Function," IEEE J. Select. Areas Commun., vol. 18, no.3, pp. 535-547, Mar. 2000. 